\title{
Serial transfer can aid the evolution of autocatalytic sets
}

Wim Hordijk', Nilesh Vaidya ${ }^{2,3}$ and Niles Lehman ${ }^{2 *}$

\begin{abstract}
Background: The concept of an autocatalytic set of molecules has been posited theoretically and demonstrated empirically with catalytic RNA molecules. For this concept to have significance in a realistic origins-of-life scenario, it will be important to demonstrate the evolvability of such sets. Here, we employ a Gillespie algorithm to improve and expand on previous simulations of an empirical system of self-assembling RNA fragments that has the ability to spontaneously form autocatalytic networks. We specifically examine the role of serial transfer as a plausible means to allow time-dependent changes in set composition, and compare the results to equilibrium, or "batch" scenarios.

Results: We show that the simulation model produces results that are in close agreement with the original experimental observations in terms of generating varying autocatalytic (sub)sets over time. Furthermore, the model results indicate that in a "batch" scenario the equilibrium distribution is largely determined by competition for resources and stochastic fluctuations. However, with serial transfer the system is prevented from reaching such an equilibrium state, and the dynamics are mostly determined by differences in reaction rates. This is a consistent pattern that can be repeated, or made stronger or weaker by varying the reaction rates or the duration of the transfer steps. Increasing the number of molecules in the simulation actually strengthens the potential for selection.

Conclusions: These simulations provide a more realistic emulation of wet lab conditions using self-assembling catalytic RNAs that form interaction networks. In doing so, they highlight the potential evolutionary advantage to a prebiotic scenario that involves cyclic dehydration/rehydration events. We posit that such cyclicity is a plausible means to promote evolution in primordial autocatalytic sets, which could later lead to the establishment of individual-based biology.
\end{abstract}

Keywords: Serial transfer, RNA, Gillespie algorithm, Origins of life, Autocatalytic set

\section{Background}

\section{Autocatalytic sets}

An autocatalytic set is a collection of molecular species in which the network as a whole forms a functionally closed and self-sustaining system [1]. This network-based perspective, as opposed to an individual-based one, is becoming recognized as a plausible early stage in the emergence of life. In the past decade, this idea has been explored formally, by casting it into graph and probability theory $[2,3]$ and examining its underlying chemical dynamics $[4,5]$, by generalizing it to other systems $[4,6]$, and by exploring the features of minimal sets that fit its definition [7]. In fact

\footnotetext{
*Correspondence: niles@pdx.edu

2Department of Chemistry, Portland State University, PO Box 751, Portland, OR 97207, USA

Full list of author information is available at the end of the article
}

the relevance of autocatalytic sets to a wide variety of phenomena is now becoming apparent [8].

Only very recently though, has progress in the laboratory in creating actual such sets with informational molecules [7-13] enabled a powerful convergence of empirical and theoretical approaches to their study. These works were inspired by earlier studies with short nucleic acid oligomers [14]. RNA, through specific nucleotide-pairing interactions that allow certain sequences to recognize each other and discriminate among similar sequences, has the ability to spontaneously form complex networks. Moreover, if these RNAs are catalytic in that they can augment the rate of synthesis of other RNAs, an autocatalytic network can be set up [10]. Previous numerical simulations of such networks have emulated well the interaction events observed in the laboratory, and furthermore confirm that cooperativity in
() Chemistry Central 
such networks can lend RNA with a competitive advantage over selfish systems that are less able or unable to form autocatalytic sets [15]. Yet the creation of true autocatalytic sets in the laboratory is still a challenging process, making it therefore essential to have more realistic and accurate computer models to make progress in this field.

Our work before has exploited the fact that the Azoarcus ribozyme, which is roughly 200 nucleotides (nt), can be broken into up to four shorter oligonucleotide fragments (termed $\mathbf{W}, \mathbf{X}, \mathbf{Y}$, and $\mathbf{Z}$ ) and that these fragments can spontaneously reassemble into covalent versions of the ribozyme. The chemistry employed for this is transesterification, which effectively means that RNA fragments can be catalytically recombined into new, larger RNAs. The assembly can happen from four pieces:

$$
\mathrm{W}+\mathrm{X}+\mathrm{Y}+\mathrm{Z} \rightarrow \mathrm{W} \cdot \mathrm{X} \cdot \mathrm{Y} \cdot \mathrm{Z}
$$

but one can also set up the reaction to occur from various combinations of only two pieces:

$$
\begin{aligned}
& \mathrm{W}+\mathrm{XYZ} \rightarrow \mathrm{W} \cdot \mathrm{XYZ} \\
& \mathrm{WX}+\mathrm{YZ} \rightarrow \mathrm{WX} \cdot \mathrm{YZ} \\
& \text { or } \mathrm{WXY}+\mathrm{Z} \rightarrow \mathrm{WXY} \cdot \mathrm{Z}
\end{aligned}
$$

In any of these cases, the dot $(\bullet)$ represents a covalent bond and the reactions are catalyzed either by noncovalent assemblages of ribozymes that form through base-paring and $3^{\circ}$ interactions or by partially or fully covalent versions of the ribozyme through autocatalytic feedback. Importantly, the specificity of these reactions is guided through specific nucleotide triplet-triplet interactions (see "The RNA system", below). These triplets can be altered in a way that rather complicated networks of RNAs can be created in which covalent assembly requires spontaneous cooperation between other sets of RNAs and the compositions of these networks can be observed to change in real time [10]. We have previously modeled some aspects of this system and found that many of its parts can be parameterized and that in general there is good correspondence between expected and observed behavior, particularly in regard to RNA-RNA cooperation dynamics [15].

An immediate requirement of both empirical and simulation studies of RNA autocatalytic sets is that they be able to accommodate evolutionary principles. These sets must be able to change their composite species frequencies over time and respond to selection pressures if they are to be relevant models of historical events on the prebiotic Earth. In principle, autocatalytic sets can evolve by expanding (including more members), contracting (expelling some members), and/or by changing the identities and relative concentrations of their composite members. Note that these evolutionary events are nonDarwinian in the traditional sense because the relative fitnesses of individual genotypes is not the underlying driving force for persistence. Nevertheless the frequency changes of network members - compositional changes with time, should they be based on selectable forces, would represent a primitive sort of evolutionary change that has closer ties to chemistry rather than biology.

These sorts of evolutionary processes are difficult to envisage in a static, or "batch", environment once the system reaches equilibrium. Thus there is a need to explore realistic models of environmental change that permit network evolution. A serial drying and rehydration of an aqueous environment is a commonly invoked feature of the prebiotic Earth [16-18] and one in which many types of prebiotic chemistry - including those associated with nucleic-acid synthesis - actually become facilitated. Here, we explore the efficacy of a common laboratory mimic of one potential consequence of dehydration/rehydration cycles, namely serial dilution, to promote the evolution of autocatalytic sets. We do this by expanding and improving the match between a previous model [15] and the chemical details of an RNA autocatalytic set [10], and simultaneously investigate the effects of serially transferring a fraction of one mixture of RNA molecules into a naïve environment containing new food-stock for RNA synthesis.

\section{The RNA system}

We based our simulations on an RNA system in which composite RNA oligonucleotides, ranging in size from $\sim 40$ $150 \mathrm{nt}$, can spontaneously self-assemble into a recombinase ribozyme [19]. These are fragments of the Azoarcus self-splicing group I intron [20], which when fully assembled through contiguous covalent bonds, is roughly $200 \mathrm{nt}$ in length. This ribozyme can catalyze further covalent synthesis of other such ribozymes, creating an autocatalytic feedback loop [21]. The initiation of this catalytic cycle is made possible by the noncovalent association of RNA fragments (here, "food" molecules) into loose coalitions that possess a small amount of activity towards recombining other fragments into covalently joined molecules [19].

Information transfer in this chemical system is mediated by a 3-nt base-pairing interaction between the internal guide sequence (IGS) located on the $5^{\prime}$ end of the ribozyme, and corresponding pseudo-complementary 3-nt "tag" sequences located on the 3' ends of each RNA fragment. In other words, catalysts (either the loose coalitions or the covalently contiguous forms of the ribozyme), can see and discriminate among other RNAs on the basis of this 3-nt interaction. To a first approximation, an IGS triplet that does not make strong Watson-Crick base pairs with a tag will not promote 
assembly using the RNA that contains that tag. Thus, interactions can be described as either "cooperative" (if an IGS base pairs with tags on other molecules with different IGS sequences) or "selfish" (if an IGS only base pairs with tags on molecules with the same IGS sequence). With this discriminatory situation, various sorts of interaction networks can be established [10]. To examine what sorts of ribozymes might emerge from a simulated pre-biotic scenario, we previously mixed fragments of these RNAs in which the middle nucleotide of the IGS (symbolized here as $\mathrm{M}$ ) and that of the tags (symbolized here as $\mathrm{N}$ ) were randomized. We were able to observe the synthesis of various frequencies of covalently contiguous full-length (200 nt) ribozymes over time in both batch (i.e., one-pot) scenarios and serial transfer scenarios in which $10 \%$ of the RNA solution was transferred to a new tube every hour [10]. For the batch scenario, the establishment of equilibrium after a few hours of reaction time revealed that cooperativity could in some cases produce higher concentrations of full-length ribozymes than selfishness, and this was corroborated and explained using ODE and stochastic simulation models $[10,15]$. However the serial transfer case was not analyzed, and the full stochastic nature of the system requires enhancements to the model. Here we explore both of these aspects.

\section{Experimental}

\section{The model}

We have constructed a formal model of the experimental RNA system and used the Gillespie algorithm to simulate its dynamical behavior. An earlier, but simpler, model was described previously [15]. Here we include more chemical realism in the model and perform a more detailed analysis. To fully specify the model, we first need to define the molecular species, the possible chemical reactions, which molecules catalyze which reactions, and the corresponding reaction rates. Then the simulation algorithm is described in detail.

\section{Molecules}

There are three main groups of molecule types. First, there are the RNA fragments (first group). These constitute the "food set" in the context of autocatalytic sets. Second, these RNA fragments associate spontaneously into non-covalent ribozymes (second group). And third, the non-covalent ribozymes are transformed (through catalyzed reactions) into the corresponding covalent ribozymes (third group).

For the purposes of the model, in the first group of molecule types (the RNA fragments), we ignore the specific junction at which the RNA fragments are covalently recombined, and only consider the particular combination of nucleotides in the IGS and tag sequences of the resulting (non-covalent) ribozyme to be important. Since the RNA fragments are oriented (left to right, or $5^{\prime}$ to $3^{\prime}$ ), we assume there is a "left" (l) fragment and a "right" (r) fragment that associate, each of which can have any of the four possible nucleotides in the IGS/tag sequence. Consequently, there are four possible "left" fragments and four possible "right" fragments, i.e., eight RNA fragments constituting the first group of molecule types (the food set). We label these $l_{M}$ and $r_{N}, M, N \in\{A, C, G, U\}$.

Given an association of such a "left" fragment and a "right" fragment, there can be 16 possible resulting noncovalent ribozymes (second group of molecule types), labeled $I_{M N}, M, N \in\{A, C, G, U\}$, depending on the relevant nucleotides $\mathrm{M}$ and $\mathrm{N}$ that are combined.

Finally, there are the 16 corresponding covalent ribozymes (third group of molecule types), labeled $E_{M N}, M$, $\mathrm{N} \in\{\mathrm{A}, \mathrm{C}, \mathrm{G}, \mathrm{U}\}$. Thus, in total there are 8 (first group) +16 (second group) +16 (third group) $=40$ molecule types in the model.

\section{Reactions}

There are two main groups of reactions. First, there are the (spontaneous) association reactions transforming two RNA fragments into a non-covalent ribozyme: $l_{\mathrm{M}}+$ $r_{N} \rightarrow I_{M N}$. There are 16 such reactions (one for each of the 16 possible non-covalent ribozymes). We also include the reverse (dissociation) reactions in the model.

Second, there are the catalyzed recombination reactions that convert a non-covalent ribozyme into a covalent one: $\mathrm{I}_{\mathrm{MN}} \rightarrow \mathrm{E}_{\mathrm{MN}}$. There are 16 such reactions (one for each of the 16 possible ribozymes), and they can be catalyzed by either a non-covalent or a covalent ribozyme (see below). We also include the reverse reactions in the model. Thus, in total there are $16+16=32$ (bi-directional) reactions in the model.

\section{Catalysis}

The ribozymes (both non-covalent and covalent) catalyze each others' transformation from non-covalent to covalent. In particular, if a ribozyme $\mathrm{E}_{\mathrm{MN}}\left(\right.$ or $\mathrm{I}_{\mathrm{MN}}$ ) has a nucleotide $\mathrm{M}$ in its guide sequence that is the base-pair complement of the variable nucleotide $\mathrm{N}^{\prime}$ in the tag sequence of another ribozyme $\mathrm{I}_{\mathrm{M}^{\prime} \mathrm{N}}$, then the first ribozyme $\mathrm{E}_{\mathrm{MN}}\left(\mathrm{I}_{\mathrm{MN}}\right)$ can catalyze the non-covalent to covalent transformation of the second ribozyme $\mathrm{I}_{\mathrm{M}^{\prime} \mathrm{N}}$. So, each ribozyme catalyzes the transformation reaction of four other ribozymes (or possibly of three others and that of itself). There is a difference in rates depending on whether the catalyst is a non-covalent $\left(\mathrm{I}_{\mathrm{MN}}\right)$ or covalent $\left(\mathrm{E}_{\mathrm{MN}}\right)$ ribozyme (see below).

The RNA fragment association reactions (forming noncovalent ribozymes) are spontaneous. In the formal autocatalytic sets framework, spontaneous (non-catalyzed) reactions can by definition never be part of an autocatalytic 
set. This is because, usually, spontaneous reactions happen at significantly lower rates than catalyzed ones, often too low to be relevant. However, in the RNA system the spontaneous RNA-RNA association reactions actually happen at very high rates because these aggregations are driven by favorable base-paring and tertiary interactions, which are numerous in the Azoarcus ribozyme [22]. In the formal framework, we can incorporate such spontaneous but high-rate reactions by assuming that they are actually catalyzed by a (fictional) "generic catalyst" which is also part of the food set. Here, this assumption is made implicitly, but not explicitly included in the model.

\section{Rates}

Relative rates for the transformation reactions were obtained experimentally by steady-state kinetic analyses of representative matching and non-matching (i.e., WatsonCrick) IGS-tag relationships in RNA fragments [10,21]. These rates were then re-scaled to make one time unit in the simulation correspond roughly to one hour in the real experiments. In these experiments, at the end of each transfer step (one hour), close to $20 \%$ of the solution has been transformed into covalent ribozymes [10]. Using this as a target, the experimentally obtained rates were then rescaled (while maintaining their relative ratios) such that after one time unit in the simulation also close to $20 \%$ of the solution consists of covalent $\left(\mathrm{E}_{\mathrm{MN}}\right)$ ribozymes.

The transformation reaction rates depend on the particular base-pair combination of the relevant nucleotide in the IGS of the catalyst and that of the tag sequence in the reactant. The following (re-scaled) rates were obtained for the four possible Watson-Crick base-pair combinations (ordered from high to low rates):

\section{AU: 0.00613, CG: 0.00541, UA: 0.00517, and GC: 0.00445.}

For example, the transformation reaction $\mathrm{I}_{\mathrm{CU}} \rightarrow \mathrm{E}_{\mathrm{CU}}$ catalyzed by the covalent ribozyme $\mathrm{E}_{\mathrm{AG}}$ has a rate of 0.00613 (catalyst/reactant base-pair combination AU).

The rates of non-covalent ribozyme catalyzed transformation reactions are set to half the rates of the corresponding covalent ribozyme catalyzed reactions, as observed in the laboratory experiments [10]. The rates of the spontaneous association reactions in the simulation model are set to 0.00006 (same rate for each of the 16 possible association reactions). Finally, the rates for all reverse reactions were set to $1 / 10$ th the rate of their corresponding forward reactions.

\section{Simulation}

To simulate the dynamics of the above chemical reaction system, we used the Gillespie algorithm [23,24] assuming a closed reaction vessel (with volume 1). Starting with an initial amount of 2000 of each of the 8 food molecule types (i.e., a total amount of 16,000 molecules), the algorithm is run for a given number of time units $t$. We performed two types of simulations: (i) with and (ii) without transfer steps.

In a simulation without transfer steps, the Gillespie algorithm is simply run for the given number of time units. In a simulation with transfer steps, at the end of each time unit first the solution is diluted to $10 \%$. Rather than simply reducing the concentration of each molecule type to $10 \%$, this dilution is done by randomly drawing molecules (without replacement) from the solution with probabilities according to their relative current concentrations, until $10 \%$ of the total concentration is reached. Next, from this diluted solution a random sample (with replacement) of 75 molecules is taken from among the $16 E_{M N}$ types. Each $E_{M N}$ type that occurs twice or more in this random sample is reported. Finally, the diluted solution is replenished with the 8 food molecule types (in equal concentrations) until the initial total concentration of 16,000 is reached again. These transfer steps are then repeated for the given number of time units. This provides a detailed simulation of the original laboratory serial transfer experiments [10], which can be repeated an arbitrary number of times.

As a consequence of computational limitations, the total amount of molecules used $(16,000)$ is relatively small, and clearly does not compare to the micromolar concentrations in the real experiments. However, to get an idea of how the system's behavior scales with larger numbers of molecules, we also performed a number of runs of the same simulation but with two orders of magnitude more food molecules (starting with 200,000 molecules of each food type, or a total of $1.6 \mathrm{E} 6$ molecules, and appropriately adjusting the time units to maintain a close to $20 \%$ conversion to $\mathrm{E}_{\mathrm{MN}}$ ribozymes after one time unit). Even though this is still a small number compared to the laboratory experiments, we argue that in an origin-of-life context the actual concentrations were most likely significantly lower than micromolar, giving more relevance to the results presented here.

\section{Results and discussion}

We first ran the simulation model without transfer steps for $t=8$ time units. Figure 1 shows the concentrations of the 16 covalent ribozymes $E_{M N}$ over time for one such run. By the end of the run, the system seems to have reached an equilibrium state. Given an initial concentration of 2000 for each of the food molecule types (RNA fragments), each $\mathrm{E}_{\mathrm{MN}}$ type can potentially reach a total concentration of 500 . However, due to the stochastic nature of the Gillespie algorithm (as in real chemistry), the RNA fragments will not associate into the intermediate non-covalent ribozymes in exactly equal amounts. There is competition among the different $\mathrm{E}_{\mathrm{MN}}$ molecules for 


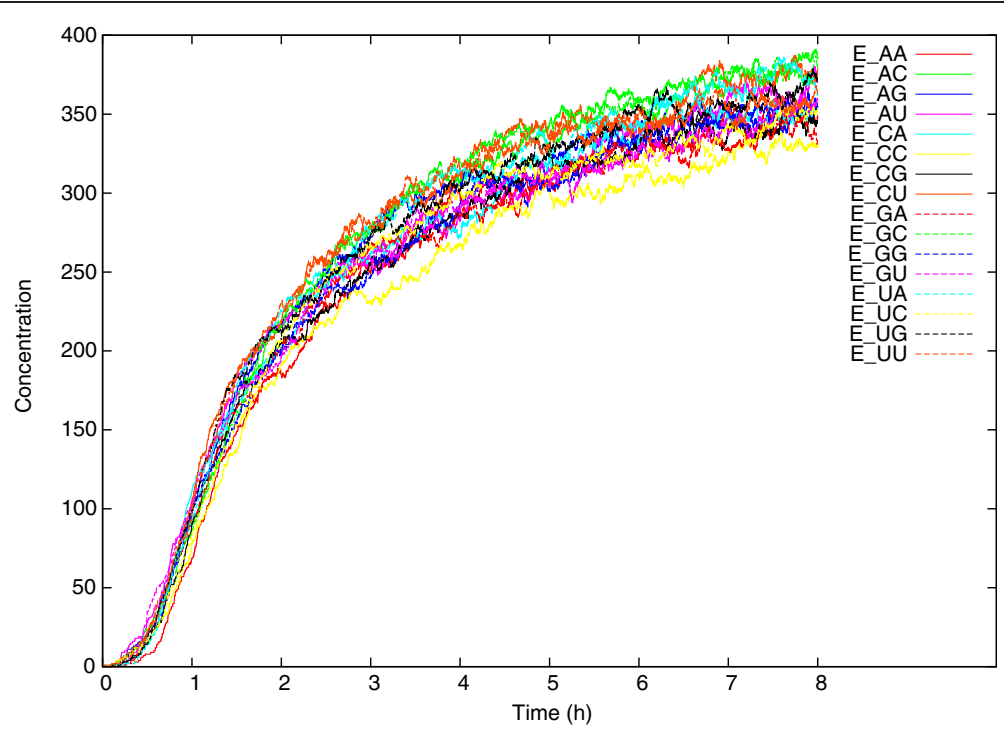

Figure 1 The concentrations of the covalent ribozymes $E_{M N}$ over time in a typical run of the simulation model (starting with 16,000 food molecules) without transfer steps, for a total of $t=8$ time units.

the same resources, and the relative concentrations in the equilibrium state are thus largely a result of stochastic fluctuations in the production of the different noncovalent ribozymes. For example, in one typical run of the simulation, the total concentrations of $\mathrm{I}_{\mathrm{MN}}+\mathrm{E}_{\mathrm{MN}}$ molecules for the 16 different types at the end of the run (eight time units) vary from 390 to 431, a difference of 41 , or around $10 \%$.

Furthermore, due to the reverse reactions [25], reflected in the wiggly lines in Figure 1, the equilibrium state is a dynamic one. Covalent ribozymes can be transformed back into non-covalent ones, which can dissociate again into RNA fragments (at a rate of $1 / 10^{\text {th }}$ of the corresponding forward reactions). As a consequence, the potential concentration of 500 is not reached and, moreover, the relative concentrations still change over time, even in the equilibrium state. Indeed, ordering the $16 \mathrm{E}_{\mathrm{MN}}$ molecule types according to their final concentrations (at $t=8$ ) gives a different (and seemingly random) order in different simulation runs.

Next, we ran the simulation model with transfer steps, also for $t=8$ time units (as in the original chemical

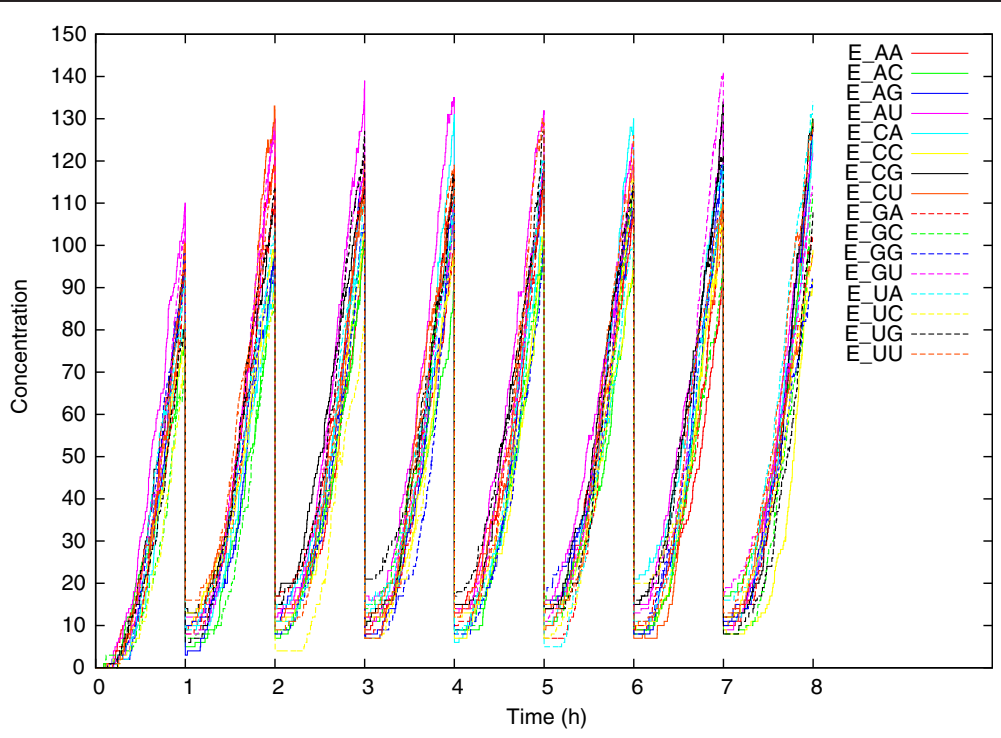

Figure 2 The concentrations of the covalent ribozymes $E_{M N}$ over time in a typical run of the simulation model (starting with 16,000 food molecules) with transfer steps, for a total of $t=8$ time units. 
experiments). Figure 2 shows the concentrations of the 16 covalent ribozymes $\mathrm{E}_{\mathrm{MN}}$ over time for one such run. The effect of the transfer steps can clearly be seen. At the end of each time unit, the concentrations are roughly around 100 (i.e., 20\% of the potential 500), which are diluted to about 10 during the transfer step. These concentrations then quickly start increasing again due to the resupply of food molecules at the end of each transfer step.

These simulation results seem to reproduce the experimental results quite well. For example, Figure 3 shows the results of taking a sample of size 75 from among the $E_{M N}$ molecules (after the dilution to $10 \%$ ) at different time steps. These graphs are indeed very similar to the experimental ones (Figure four in ref [10]). Also, they confirm the previous model prediction [15] that a different sequence of networks is observed each time the experiment/ simulation is repeated, due to the stochastic nature of the system.

The transfer steps clearly prevent the system from reaching an equilibrium state. It turns out that this has an important impact on the relative concentrations of the covalent ribozymes. Whereas these relative concentrations in the equilibrium state are largely a consequence of competition for resources (as explained above), on short time scales, when there are still abundant food molecules available, they are largely determined by differences in the reaction rates.

This distinction is shown in Table 1 . The first column shows the average concentrations of the covalent ribozymes (starting with 16,000 food molecules and averaged over 50 simulation runs) after one time unit $(t=1)$, and ordered from highest to lowest. The second column shows the same but after eight time units $(t=8$; no transfer steps performed). Clearly, after only one time unit, the covalent ribozymes that have the highest rate
Table 1 Short-term vs long-term dynamics

\begin{tabular}{|c|c|c|c|c|c|c|c|}
\hline \multicolumn{4}{|c|}{16,000 food molecules } & \multicolumn{4}{|c|}{$1,600,000$ food molecules } \\
\hline \multicolumn{2}{|c|}{$t=1$} & \multicolumn{2}{|c|}{$t=8$} & \multicolumn{2}{|c|}{$t=1$} & \multicolumn{2}{|c|}{$t=8$} \\
\hline UU & 101.1 & CA & 365.0 & $\mathrm{CU}$ & 9899 & CG & 36200 \\
\hline$A U$ & 100.8 & AG & 364.8 & UU & 9888 & UA & 36160 \\
\hline CU & 99.0 & GU & 363.4 & GU & 9875 & $\mathrm{AU}$ & 36145 \\
\hline GU & 98.5 & GG & 363.3 & $\mathrm{AU}$ & 9873 & GU & 36139 \\
\hline AG & 96.3 & UU & 362.1 & AG & 9290 & GG & 36121 \\
\hline CA & 94.3 & $A C$ & 361.4 & GG & 9276 & $\mathrm{CU}$ & 36116 \\
\hline UG & 92.5 & AA & 360.3 & UG & 9272 & UG & 36112 \\
\hline GA & 91.8 & GC & 360.2 & CG & 9268 & $\mathrm{AA}$ & 36094 \\
\hline GG & 91.5 & CG & 360.1 & CA & 9054 & $A C$ & 36092 \\
\hline AA & 90.3 & UG & 359.8 & $\mathrm{AA}$ & 9043 & GA & 36083 \\
\hline UA & 90.1 & CC & 359.6 & $\mathrm{GA}$ & 9022 & $C A$ & 36082 \\
\hline CG & 90.0 & $\mathrm{AU}$ & 359.6 & UA & 9017 & UC & 36072 \\
\hline$A C$ & 83.9 & UC & 359.2 & GC & 8305 & $A G$ & 36069 \\
\hline GC & 83.7 & UA & 358.7 & CC & 8302 & CC & 36059 \\
\hline CC & 82.5 & GA & 358.6 & UC & 8300 & GC & 36054 \\
\hline UC & 81.6 & $C U$ & 357.1 & $A C$ & 8257 & UU & 36053 \\
\hline
\end{tabular}

The average number of molecules of the 16 covalent ribozymes after one time unit $(t=1)$ and eight time units $(t=8)$ without transfer steps, ordered from high to low. The left half of the table is for simulations starting with 16,000 food molecules (2,000 of each type), averaged over 50 runs. The right half of the table is for simulations starting with $1,600,000$ food molecules $(200,000$ of each type), averaged over 20 runs.

of production (the $E \mathrm{U}$ molecules with rate 0.00613 ) also have the highest concentrations on average (first four molecule types in the first column). Furthermore, the ribozymes with the lowest rate of production (the $\mathrm{E}^{{ }^{*} \mathrm{C}}$ molecules with rate 0.00445 ) have the lowest average concentration (last four molecule types in the first column). The other two molecule types ( $\mathrm{E}_{*_{\mathrm{A}} \mathrm{A}}$ and $\left.\mathrm{E}_{*_{\mathrm{G}}}\right)$, which have similar rates in between $(0.00517$ and 0.00541 , respectively), are mixed and between the $E_{* U}$

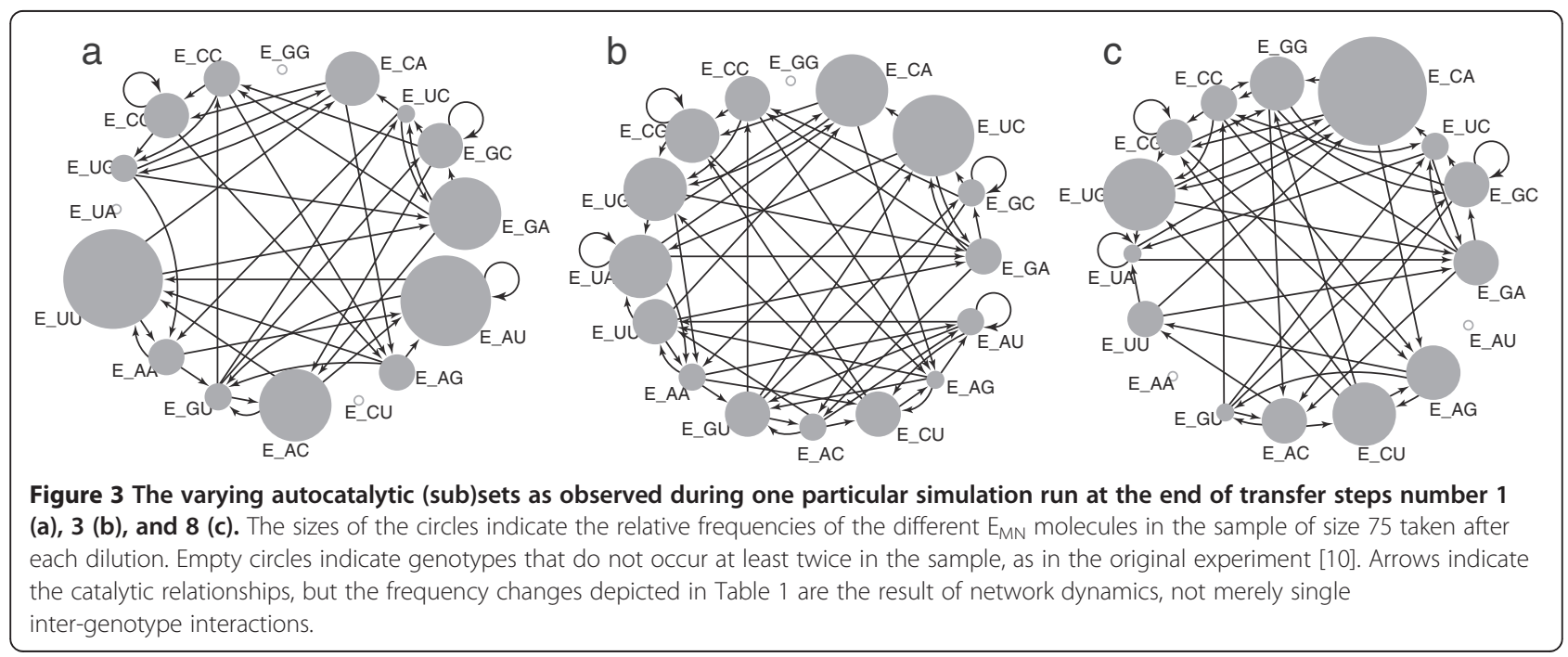


and $E^{*} \mathrm{C}$ molecules. This pattern is the same on any set of 50 runs over which the averages are calculated (although the order of the four $\mathrm{E} \mathrm{U}$ molecule types within the top four might be different, and similarly for the order of the four $E^{{ }^{*} \mathrm{C}}$ molecule types within the bottom four).

However, after eight time units the situation is very different, as already observed earlier. There is no clear pattern in the ordering (second column) and, in fact, the ordering is completely different (and seemingly random) over different sets of 50 runs. Furthermore, the variance in the average concentrations is much lower. After one time unit, the difference between the highest and lowest concentrations is $101.1-81.6=19.5$ (which is $19.3 \%$ of the highest concentration). However, after eight time units, this difference is $365.0-357.1=7.9$ (which is only $2.2 \%$ of the highest concentration). So, not only in absolute value, but also in relative value the variance in concentrations is much higher after only one time unit than after eight time units.

This pattern of the relative concentrations being largely determined by the differences in reaction rates is still clearly visible after two time units (no transfer steps) as well, although with a lower variance in concentrations (9.84, or $4.6 \%$ of the highest concentration). However, after four time units (no transfer steps), the pattern has already disappeared, and the ordering is more or less random and the variance very low, just as after eight time units. This pattern can be made even stronger by making the reaction rates even more different. For example, increasing the rate of production of $E^{*} \mathrm{U}$ molecules and decreasing the rate of production of $\mathrm{E}^{{ }^{*} \mathrm{C}}$ molecules, the ordered pattern can persist even up to three or four time units (no transfer steps). Obviously if the production rates of all $\mathrm{E}_{\mathrm{MN}}$ molecules are equal, than the ordering is always random, even already after one time unit.

Now, measuring the relative concentrations of the covalent ribozymes at the end of the simulation run over eight time units and with transfer steps, again averaged over 50 runs, shows the same ordered pattern as the first row of Table 1. As mentioned, the transfer steps prevent the system from reaching the (random) equilibrium state and, as a consequence, the relative concentrations are still largely determined by the differences in reaction rates, not by competition for resources. This pattern is the same, no matter how many transfer steps are performed, as long as the duration of the transfer steps is relatively short (on the order of one or two time units). Note that the concentrations at the end of any one particular transfer step do not necessarily reflect this ordered pattern (cf. Figure 3). However, on average (over a larger number of transfer steps), this pattern is clear and consistent.

To see whether this phenomenon is not an artifact of the relatively small number of molecules used in the simulations, we repeated the analysis with a starting concentration of 1,600,000 food molecules $(200,000$ of each type), and averaging over 20 runs. The results are shown in the third $(t=1)$ and fourth $(t=8)$ columns of Table 1. Clearly, the effect is even stronger in this case, even though the averages are over a smaller number of runs (20 instead of 50, due to higher computational requirements). Now also the two "intermediate" ribozyme types $\left(E_{*^{*} \mathrm{~A}}\right.$ and $\left.E_{*_{\mathrm{G}}}\right)$ are well separated after one time unit. So, we can expect that with even larger concentrations, the effect on selection is even stronger.

\section{Conclusions}

We have improved and expanded our previous model [15] of an autocatalytic set that is observed in a selfassembling RNA system that is a plausible mimic of the origins of information transfer. Our main observation is that serial transfer has the effect of preventing the molecular system from reaching chemical equilibrium, thereby augmenting the power of slight differences among rate constants to promote selection. While a batch system can generate different frequencies of its final products, in the context of network dynamics, the evolutionary potential of such a scenario is highly limited. With serial transfer, we can observe changes in network composition over time (Figure 3) that are dependent and driven by relatively small intrinsic variations in 3-nt base-pairing interactions, whose free energies can differ by less than $1 \mathrm{kcal} / \mathrm{mole}$ in the active site of catalytic RNAs such as the ones under study [26]. Our results were obtained even when the composition of the food-stock was kept constant in each transfer; true environmental systems would not be so regular and the effects of serial transfer on selection should be similarly augmented. This phenomenon should be applicable to a wide range of autocatalytic set realizations including protein networks [11], nucleic-acid networks $[9,10]$, and lipid networks such as the composomes envisioned in the GARD model of Lancet and coworkers [27]. We posit that a pre-biotic scenario of dehydration/rehydration would be permissive for the evolution of autocatalytic networks and thus should be explored further.

\section{Competing interests}

The authors declare that they have no competing interests.

\section{Authors' contributions}

WH developed the simulation model, NV and NL helped analyze the simulation results, and $\mathrm{WH}$ and NL wrote the paper. All three authors read and approved the final manuscript.

\section{Acknowledgements}

This work was supported by a grant from the National Aeronautics and Space Administration (NNX10AR15G to N.L.). 


\section{Author details}

${ }^{1}$ SmartAnalytiX.com, Lausanne, Switzerland. '2Department of Chemistry, Portland State University, PO Box 751, Portland, OR 97207, USA. ${ }^{3}$ Present address: Department of Chemical and Biological Engineering, Princeton University, Princeton, NJ 08544, USA.

Received: 18 December 2013 Accepted: 12 March 2014

Published: 26 April 2014

\section{References}

1. Kauffman SA: The Origins of Order. New York: Oxford University Press; 1993.

2. Hordijk W, Steel M: Detecting autocatalytic, self-sustaining sets in chemical reaction systems. J Theor Biol 2004, 227:451-461.

3. Mossel E, Steel M: Random biochemical networks: the probability of self-sustaining autocatalysis. J Theor Biol 2005, 233:327-336.

4. Hordijk W, Steel M: Autocatalytic sets extended: dynamics, inhibition, and a generalization. J Syst Chem 2012, 3:5

5. Vasas V, Fernando C, Santos M, Kauffman SA, Szathmáry E: Evolution before genes. Biology Direct 2012, 7:1.

6. Hordijk W, Steel M, Kauffman SA: The structure of autocatalytic sets: Evolvability, enablement, and emergence. Acta Biotheoretica 2012, 60:379-392.

7. Steel M, Hordijk W, Smith J: Minimal autocatalytic networks. J Theor Biol 2013, 332:96-107.

8. Hordijk W: Autocatalytic sets: from the origin of life to the economy. BioScience 2013, 63:877-881.

9. Lincoln TA, Joyce GF: Self-sustained replication of an RNA enzyme. Science 2009, 323:1229-1232.

10. Vaidya N, Manapat ML, Chen IA, Xulvi-Brunet $R$, Hayden EJ, Lehman N: Spontaneous network formation among cooperative RNA replicators. Nature 2012, 491:72-77.

11. Ashkenasy $G$, lagasia R, Yadav M, Ghadiri MR: Design of a directed molecular network. Proc Natl Acad Sci USA 2004, 101:10872-10877.

12. Qian L, Winfree E, Bruck J: Neural network computation with DNA strand displacement cascades. Nature 2011, 475:368-372.

13. Yin $P$, Choi HMT, Calvert CR, Pierce NA: Programming biomolecular self-assembly pathways. Nature 2008, 451:318-322.

14. Sievers D, von Kiedrowski G: Self-replication of complementary nucleotide-based oligomers. Nature 1994, 369:221-224.

15. Hordijk W, Steel M: A formal model of autocatalytic sets emerging in an RNA replicator system. J Syst Chem 2013, 4:3.

16. Kuhn $\mathrm{H}$ : Model consideration for the origin of life. Environmental structure as stimulus for the evolution of chemical systems. Naturwissenschaften 1976, 63:68-80

17. Lahav N, White D, Chang S: Peptide formation in the prebiotic era: thermal condensation of glycine in fluctuating clay environments. Science 1978, 201:67-69.

18. Deamer D: First Life: Discovering the Connections between Stars, Cells, and How Life Began. Berkeley: University of California Press; 2012.

19. Hayden EJ, Lehman N: Self-assembly of a group I intron from inactive oligonucleotide fragments. Chem Biol 2006, 13:909-918.

20. Reinhold-Hurek B, Shub DA: Self-splicing introns in tRNA genes of widely divergent bacteria. Nature 1992, 357:173-176.

21. Hayden EJ, von Kiedrowski G, Lehman N: Systems chemistry on ribozyme self-construction: Evidence for anabolic autocatalysis in a recombination network. Angew Chem Int Ed 2008, 47:8424-8428.

22. Adams PL, Stahley MR, Kosek AB, Wang J, Strobel SA: Crystal structure of a self-splicing group I intron with both exons. Nature 2004, 430:45-50.

23. Gillespie DT: A general method for numerically simulating the stochastic time evolution of coupled chemical reactions. J Comput Phys 1976, 22:403-434

24. Gillespie DT: Exact stochastic simulation of coupled chemical reactions. J Phys Chem 1977, 81:2340-2361

25. Vaidya N, Walker SI, Lehman N: Recycling of informational units leads to selection of replicators in a prebiotic soup. Chem Biol 2013, 20:241-252.
26. Zaug AJ, Dávila-Aponte JA, Cech TR: Catalysis of RNA cleavage by a ribozyme derived from the group I intron of Anabaena pre-tRNA ${ }^{\text {Leu }}$ Biochemistry 1994, 33:14935-14947.

27. Segre D, Ben-Eli D, Lancet D: Compositional genomes: Prebiotic information transfer in mutually catalytic noncovalent assemblies. Proc Natl Acad Sci USA 2000, 97:4112-4117.

doi:10.1186/1759-2208-5-4

Cite this article as: Hordijk et al: Serial transfer can aid the evolution of autocatalytic sets. Journal of Systems Chemistry 2014 5:4

\section{Publish with ChemistryCentral and every scientist can read your work free of charge \\ "Open access provides opportunities to our colleagues in other parts of the globe, by allowing anyone to view the content free of charge." \\ W. Jeffery Hurst, The Hershey Company.}

- available free of charge to the entire scientific community

- peer reviewed and published immediately upon acceptance

- cited in PubMed and archived on PubMed Central

- yours - you keep the copyright

Submit your manuscript here:

http://www.chemistrycentral.com/manuscript/<smiles>c1ccccc1</smiles>

ChemistryCentral 